\title{
Study the Effects of Photon Radiation on Power MOSFET for Harsh Environment Application
}

\author{
Erman Azwan ${ }^{1, *}$, Ramani Kannan ${ }^{1}$, Lini Lee ${ }^{2}$, and Saranya Krishnamurthy ${ }^{1}$ \\ ${ }^{1}$ Department of Electrical and Electronic Engineering, Universiti Teknologi PETRONAS, Malaysia \\ ${ }^{2}$ Faculty of Engineering, Multimedia University, Cyberjaya, Malaysia
}

\begin{abstract}
The rapid growth of the advanced technologies in power electronics system gives a challenge to the electronic device to sustain with the modern technologies nowadays. The challenges are also including the place where the system was installed for example the application in the harsh environment. Harsh environment application requires an electronic device deals with radiation pollution. Hence, the electronic device will suffer from this phenomenon and make the whole system to malfunction and break down. Power Metal Oxide Semiconductor Field Effect Transistor (MOSFET) is one type of electronic device that is the most broadly for high voltage and high switching speed application. The aim of this paper is to studies the photon radiation effect toward the Power MOSFET performance. The study focus on the changing of the electrical characteristics of the device after radiated with photon radiation. Process simulation and Device simulation tools in Sentaurus Synopsys Software used for the research to validate all the theory.
\end{abstract}

\section{Introduction}

The implementation of Power MOSFET in harsh environment application has exposed the device to radiation pollution. Harsh environment was defined as the place or situation where all the living thing cannot be survived and sustained. There is a lot of pollution in this place and this is very critical for all electronic device to operate normally. Radiation was defined as a radioactive element that is structurally unstable and to return to a more stable, it will emit particles and energy. Theoretically, radiation is categorized into two which is photon radiation and particle radiation. Photon radiation consists of $\gamma$ rays or $x$-rays and meanwhile, particle radiation consists of charged particles such as protons, $\alpha$ particles, ions, and neutron particles [1]. The main difference between these two types of radiation is the mass and energy. Particle radiation have a mass and energy while photon radiation does not mass have but still will affect the electronic device if the range of ionization is enough to make the device to malfunction [2].

Other than that, the effect of radiation on power electronic devices that can be categorized into two type; cumulative effect and Single Event Effect (SEE). Cumulative effect means the radiation exposure will take some time to make the device degradation and it will calculate

\footnotetext{
* Corresponding author: erman.azwan@gmail.com
} 
based on the Total Ionizing Dose (TID) of the radiation. SEE is the effect that instantaneously happened once radiation particle emitted to the device if the amount of radiation is enough to make the device degradation [3]. SEE can be divided into two type which is Single Event Burnout (SEB) and Single Event Gate Rupture (SEGR). SEB will cause the high saturation of drain current in the device and potentially will burn out the device and SEGR breaks the gate structure of the Power MOSFET and will fluctuate the value of gate voltage and make the device malfunction $[3,4]$.

As aforementioned, Power MOSFET was the widely electronic devices used in many power electronic application because of the capability to work at high frequency and high voltage application. Unfortunately, the implementation of Power MOSFET in harsh environment give a big challenge for this device to maintain their performance in this crucial zone. The main issue in this scenario is the changing of device behavior once the device was introduced to radiation pollution then will affect the whole power electronic system. This paper will investigate the effect of photon radiation on Power MOSFET by using gamma-ray radiation mechanism.

\section{Radiation Mechanism}

\subsection{Photon Radiation}

There are three form of energy that is transmitted through radiation; electromagnetic waves, rays, and particles. When the radiation happens, the electrically charged particle that travels through empty space will form an electromagnetic wave and this energy can be categorized into waves and photon. Photon radiation is a massless particle but the highest energy because it has the shortest wavelength and can travel at the speed of light. This energetic photon potentially will give a significant effect to the device and the whole system. Gamma-ray is a photon radiation in electromagnetic energy that emitted by the nucleus of some radionuclides following radioactive decay. They are the most energetic photon in the electromagnetic spectrum and their emission can occur less than a second $[5,6]$.

\subsection{Radiation Effect on Power MOSFET}

Power electronics application in the harsh environment has exposed the electronic device to a catastrophic failure mechanism because of radiation pollution. Most of the device will suffer from SEE phenomena either SEGR or SEB [6]. SEGR is the phenomena that will damage the gate oxide structure by damaging the function of the gate to control the current from source to drain. If the radiation was penetrated perpendicularly with the gate oxide structure, it enhances dielectric breakdown. When the bias is applied to the gate, radiation particle will accumulate at the $\mathrm{Si} / \mathrm{SiO}_{2}$ interface and form a conduction bridge between gate and drain. This will result in a high electric field stress at the gate oxide and localizing gate rupture indirectly make dielectric breakdown. Besides, SEGR gives a great impact to the device parameter especially the threshold voltage $\left(\mathrm{V}_{\text {th }}\right)$ and drain-source voltage $\left(\mathrm{V}_{\mathrm{DS}}\right)$. The change in the drain-source resistance shifts the value of $V_{\text {th }}$ and the increase in the $V_{D S}$ [6-8].

Other than that, SEB is the phenomena that build up the permanent pathway from the source terminal to drain terminal. Radiation particle will be turned on parasitic BJT inherent in the device structure and caused a high transient current. This is because the generation of the electron-hole pair in collector region of parasitic BJT will make increase the collector currents in the BJT until the device breakdown. Moreover, in term of the device parameter, SEB will affect the drain-source current ( $\left.I_{D S}\right)$ during in off state operation. It will not affect any physical damage to the device, but the value $\mathrm{I}_{\mathrm{DS}}$ will increase due to the short circuit 
between source and drain. In addition, SEB gives more impact to p-channel MOSFET than n-channel MOSFET. In p-channel MOSFET, the magnitude of avalanche generated current is lower due to the less impact ionization rate of hole compares to electron [6-8].

\section{Simulation Description}

\subsection{Power MOSFET Design}

Power MOSFET design usually in vertical structure as to reduce the device stress during operation. The first generation of MOSEFT was invented at the beginning of the 1980s, and the main structure of the Power MOSFET was deserted in favor of Vertical Diffused MOS (VDMOS) or DMOS. The basic component of Power MOSFET are the gate, drain, source and the MOS structure is the most important part. In addition, an n-drift layer located in the substrate as to enhance the device operation performance [9]. This study will cover simulation work including designing the geometrical and materials properties of commercial Power MOSFET by using Synopsys Technology Computer Aided Design (TCAD) mixed simulation. In TCAD Synopsys, Sentaurus Structure Editor (SDE) tool used for this process and connected with Graphical User Interface (GUI) to input process step such as oxidation, deposition, epitaxy, etch and implantation. After finished designing part, the created structure will be linked to Sentaurus Device (SDevice) to perform the device simulation. SDevice can read the structure by structure grid file and parameter files. At this time, SDevice can start device simulation based on physic model listed in command.

\subsection{Reliability Test- Gamma-ray radiation}

In the device simulation, gamma-ray has used as the radiation source and all the parameter of radiation such as dose rate and dose time were set in the physic section and activated by specifying the keyword 'Radiation' as shown in Fig. 1.

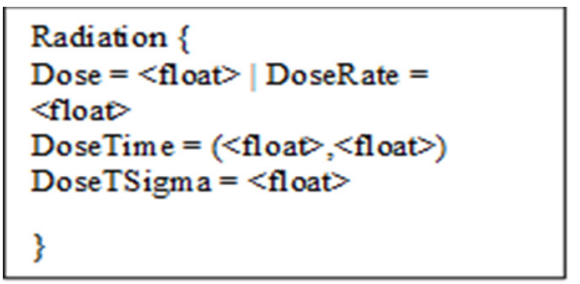

Fig. 1. The structure of coding in the physic section for radiation setup.

Based on the figure, the value of the dose rate must be calculated by using the formula in Eq. 1 and the unit rad/s. Next, the period of radiation occurs need to be decided in the optional Dose Time (s). Then, DoseTSigma (s) and Dose Time will combine to specify the standard deviation of a Gaussian rise and fall of the radiation exposure. Besides, Dose Rate (in rad) can be set as to represent the total radiation exposure over the Dose Time interval. In this case, Dose Time need to prescribe. To plot the generation rate due to gamma radiation, specify 'Radiation Generation' in the plot section [10,11].

$$
\begin{aligned}
& G_{r}=g_{0} D \times Y(F) \\
& Y(F)=\left(\frac{F+E_{0}}{F+E_{1}}\right)^{m}
\end{aligned}
$$


$\mathrm{D}$ is the dose rate, $\mathrm{g}_{0}$ is the electron-hole pairs generation rate while $\mathrm{E}_{0}, \mathrm{E}_{1}$, and $m$ are constants.

\section{Result And Discussion}

During gamma-ray radiation, two variables are used to vary the result of the research. The experiment focuses on dose time and dose rate (Linear Energy Transfer (LET)). Fig. 2 and Fig. 3 shows the electric field density for the device before and after radiation with gammaray at a dose rate of $25 \mathrm{MeV}$. Based on the figure, the device that radiated with gamma-ray has higher electrical field density especially at the gate oxide structure ( $\mathrm{Si} / \mathrm{SiO} 2$ interface) near to JFET region and it gives more impact if higher bias is applied to the gate oxide terminal. The higher density of electric field caused by the accumulation of secondary electron-hole pair in the $\mathrm{Si} / \mathrm{SiO} 2$ interface. These charges can perturb the normal operation of the device. The JFET region and gate oxide have a higher electric field when the device is on-state because the electron form depletion area to transfer the charge from source to the drain. From the obtained result, the gamma-ray radiation has brought some physically effect on the electric field distribution at the critical part of devices.

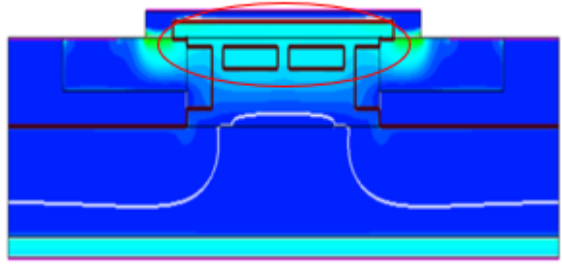

Fig. 2. Electrical field on the Virgin device.

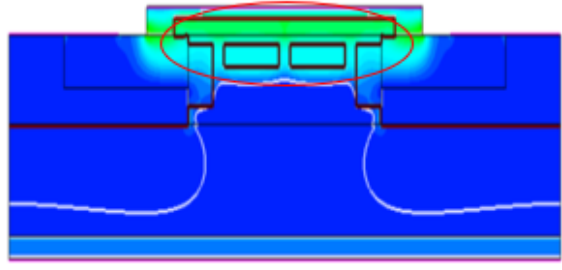

Fig. 3. Electrical field on the radiated device.

Besides, the gamma-ray also have affected the electrical characteristic of the power MOSFET. Fig. 4 and Fig. 5 shows the result of the electrical characteristic with a different variable. During gamma-ray radiation, two variable is used to vary the result of the research. The experiment focuses on dose time and dose rate (Linear Energy Transfer). The value of dose time used is in the range of $1 \mathrm{e}-3 \mathrm{sec}$ to $100 \mathrm{e}-3 \mathrm{sec}$ and the dose rate used is around 0 $\mathrm{MeV}$ to $100 \mathrm{MeV}$.

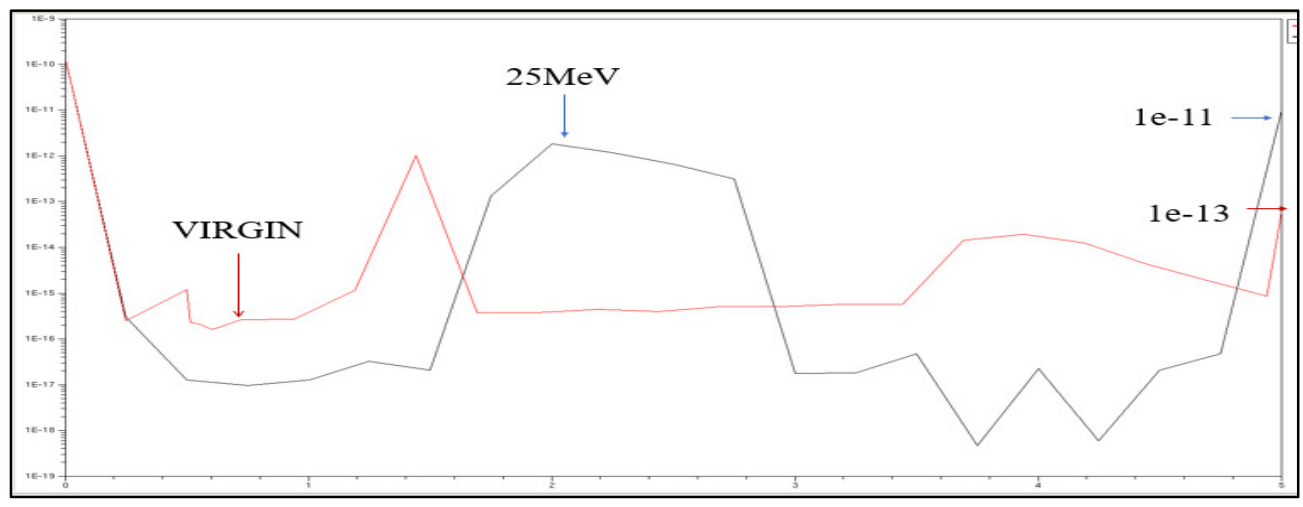

Fig. 4. Graph of Ids vs Vds for time-based study. 


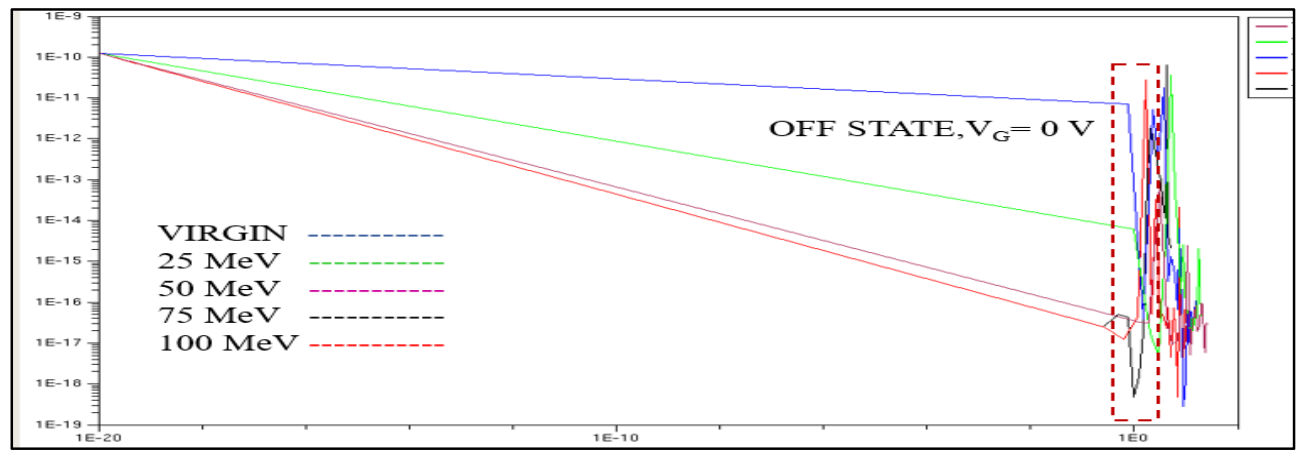

Fig. 5. Graph of IDS vs VDS for dose rate (LET-based study).

Based on Fig. 4, the period of exposure is around 1e-3 sec. From the result, a drastic effect of radiation exposure on the value of drain-source current $\left(\mathrm{I}_{\mathrm{DS}}\right)$ vs drain-source voltage $\left(\mathrm{V}_{\mathrm{DS}}\right)$. The red color line represents the virgin MOSFET and the blue color represent the device with the radiation. From the obtained result, the value of $\mathrm{I}_{\mathrm{DS}}$ is somehow shifted from $1 \mathrm{e}-13 \mathrm{~V} / \mathrm{cm}$ (Virgin) to more than $1 \mathrm{e}-11 \mathrm{~V} / \mathrm{cm}$ after the radiation exposure during the onstate region at $\mathrm{V}_{\mathrm{G}}=5 \mathrm{~V}$. Since, SEE is an instantaneous effect, hence no matter how long the time of radiation penetration onto the device it still will affect the device performance if the value of radiation energy is enough. If the device keeps operating SEB phenomena will occur to the device because the higher electric field density will be formed due generation of electron-hole pair. The generated electron-hole pair will accumulate at the insulating layer and will form a pathway (short circuit) between drain and source and cause the gate-source current ( $\mathrm{I}_{\mathrm{GS}}$ ) to increase. Lastly, the $\mathrm{I}_{\mathrm{DS}}$ also increased due to changing of drain-source resistance $\left(R_{D S}\right)$ value during on-state.

For linear energy transfer, Fig. 5 shows the graph of $I_{D S} v s V_{D S}$ for the dose rate of $0 \mathrm{MeV}$ (Virgin), $25 \mathrm{MeV}, 50 \mathrm{MeV}, 75 \mathrm{MeV}$, and $100 \mathrm{MeV}$. From the Fig. 3, the gamma-ray radiation has changed the electrical behavior of the device. There are also slightly increment on the electric field inside the device especially in the JFET region and the pathway toward the drain terminal. Result in Fig. 5 shows that the blue color line represents the virgin device and other colors line represent the device that been penetrated to the specific value of dose rate. From the graph, there is a significant effect on the value of $I_{D S}$ and it varied based on the dose rate level. The higher value of the dose rate will result the higher shifting in $\mathrm{I}_{\mathrm{DS}}$ value during the off-state region $\mathrm{V}_{\mathrm{G}}=0 \mathrm{~V}$.

\section{CONCLUSIONS}

As a conclusion, this technical paper studied the effect of photon radiation on the commercial Power MOSFET by using software simulation. The photon radiation that used in this simulation is gamma ray. From the study, the radiation exposure toward electronic device will tend the device to malfunction. From the time-based study, it shows that even though the time for radiation penetration on the device is short (microsecond) but it still gives a great impact to the device if the radiation energy achieves the breakdown potential value. Besides, for dose rate (LET-based study), the change of I-V characteristic is depending on the value of LET. The higher the LET, the higher the shifting in $\mathrm{I}_{\mathrm{DS}}$. Means that, it will give more impact to the device performance. 
The authors are thankful to the Fundamental Research Grant Scheme (FRGS 2016) with the title "A Fundamental Investigation of Radiation Effect on Metal Oxide Semiconductor Field Effect Transistor on Harsh Environment Application".

\section{References}

1. Y. H. Lho, K. Y. Kim, ETRI, 27, 449 (2005)

2. G. F. Knoll, Radiation detection and measurement (John Wiley \& Sons, 2010)

3. A. Akturk, R. Wilkins, J. McGarrity, B. Gersey, IEEE Tran. Nuc. Sci. , 64, 529 (2017)

4. A. Akturk, J. McGarrity, R. Wilkins, A. Markowski, B. Cusack, IEEE REDW, (2017)

5. M. H. Wong, A. Takeyama, T. Makino, T. Ohshima, K. Sasaki, A. Kuramata, S. Yamakoshi, M. Higashiwaki, IEEE DRC, (2017)

6. E. A. Yahya, R. Kannan, Z. Baharudin, S. Krishnamurthy, IEEE ROMA, (2017)

7. A. F. Witulski, D. R. Ball, K. F. Galloway, A. Javanainen, J. Lauenstein, A. L. Sternberg, R. D. Schrimpf, IEEE Tran. Nuc. Sci, 65, 1951 (2018)

8. C. K. Maiti ; T. P. Dash, IEEE DevIC, (2017)

9. Zhang, Qingchun, B. Hull, U.S. Pat. No. 9,530,844. 27, (2016)

10. Synopsys, I. Sentaurus Structure Editor User Guide (2013).

11. J. Kim, G. Kim, H. Nam, C. Shin, J. Park, J. Kim, B. J. Cho, K. C. Saraswat, H. Yu, IEEE Elec. De. Lett. 35, 1185 (2014) 